\title{
Expectativas del turista con el servicio de alimentos y bebidas en Cuba.
}

\author{
Expectations of the tourist with the food and drink service in Cuba.
}

\author{
Beatriz Romaní Bendig. ', Julia María Espinosa Manfugás. ${ }^{2}$, René Tejedor $\operatorname{Arias}^{3}$ \& \\ Lideimys Marieta Mesa Ramos. ${ }^{4}$
}

\section{Resumen.}

El presente trabajo tuvo como objetivo determinar las expectativas del turista con el servicio de alimentos y bebidas en el destino Cuba. Para esto se aplicó el cuestionario propuesto por Romaní, [et. al], 2018. El análisis de los datos incluyó el cálculo de porcentajes, la fiabilidad del constructo por medio del Alfa de Cronbach, el test bidimensional de Friedman y prueba de Wilcoxon para determinar las prioridades de los clientes a la hora de seleccionar un restaurante, así como el diagrama de Pareto. Los datos se procesaron mediante el programa estadístico "Statistic Program for Social Sciences" para Windows (versión 22.0). Los resultados demostraron que los indicadores más importantes a la hora de seleccionar un restaurante son la comida, la seguridad y el precio; y que las expectativas vitales sobre los servicios adicionales son música agradable, atención al cliente, información sobre la especialidad de la casa, internet y animación.

Palabras claves: expectativas, alimentos y bebidas, confiabilidad.
Abstract.
The goal of this work was to determine the tourist's expectations about the food and beverage service in the destination of Cuba. In order to do it, the questionnaire proposed by Romaní, [et. al], 2018 was applied. The analysis of the data included the calculation of percentages, the reliability of the construct by means of the Cronbach's Alpha, the Friedman two-dimensional test and the Wilcoxon test to determine the priorities of clients when selecting a restaurant, and to conclude, the Pareto Diagram. The data was processed

\footnotetext{
${ }^{1}$ Facultad de Turismo de la Universidad de la Habana, Cuba, bea_romani@ftur.uh.cu

${ }^{2}$ Facultad de Turismo de la Universidad de la Habana.Cuba, julia_espinosa@ftur.uh.cu

${ }^{3}$ Instituto Farmacia-Alimentos de la Universidad de la Habana. Cuba, tejedor@ifal.uh.cu

${ }^{4}$ Aeropuerto Internacional José Martí. Cuba.
} 
using "Statistic Program for Social Sciences" for Windows (version 22.0). The results showed that the most important indicators when selecting a restaurant are food, security and price; and that the vital expectations on the additional services are pleasant music, customer service, information about the specialty of the house, internet and animation.

Keywords: expectations, food and beverages, reliability.

\section{Introducción.}

El turismo ha sido uno de los sectores de mayor crecimiento e impacto en el siglo XX, tendencia que se consolida en el momento actual. Según el último informe de la Organización Mundial del Turismo (2017) las llegadas de turistas internacionales registraron un notable aumento del 7\% en 2017 hasta alcanzar un total de 1.322 millones. Se estima que las llegadas de turistas internacionales crezcan un 3,3\% hasta el 2030 a nivel mundial, para alcanzar en ese año 1.800 millones [1].

En el caso de Cuba, el turismo constituye una de las principales ramas de la economía que mayores ingresos han generado al país, de ahí la necesidad de brindar productos y servicios con mayor calidad, que satisfagan las expectativas de los clientes, para así incrementar la competitividad del destino Cuba.

A decir de Mesa (2017) la restauración, componente esencial del turismo, ha evolucionado en el transcurso del tiempo hasta convertirse hoy en una actividad donde prima la creatividad y la imaginación. La exquisitez de los platos, la calidad de los servicios, el valor añadido al producto gastronómico; se convierten en elementos distintivos de la competencia y son los elementos claves para lograr la satisfacción de los clientes [2].

Calás (2014) plantea que es un imperativo garantizar una oferta gastronómica acorde a las exigencias para satisfacer o superar las expectativas de los clientes y crear en ellos experiencias inolvidables [3].

Tomando en consideración que en la satisfacción final del cliente uno de los elementos más importantes a tener en cuenta son sus expectativas, se hace necesario, entonces, el desarrollo de estudios encaminados a su determinación y conocimiento, para así poder satisfacerlas.

En Cuba se han realizado numerosos trabajos enfocados a medir la satisfacción de los clientes con los servicios turísticos de manera general y de alimentos y bebidas en particular. Moreno [et. al] (2012) señala que estos últimos han sido históricamente uno de las que ha reportado mayor cantidad de insatisfacciones por parte de los clientes [4].

Sin embargo no se dispone en la actualidad de información obtenida a través de métodos científicos sobre las expectativas de los turistas con el servicio de alimentos y bebidas. Considerando la importancia que tiene el conocimiento de las expectativas del cliente en el fenómeno de la satisfacción y la necesidad de elevar sus niveles en la actividad de alimentos y bebidas, se realizó esta investigación que tiene como objetivo general determinar las expectativas del turista con el servicio de alimentos y bebidas en el destino Cuba. 


\section{Métodos.}

Para la determinación de las expectativas del turista con el servicio de alimentos y bebidas se aplicó la encuesta propuesta por Romaní, [et. al], 2018, según se reporta, se determinó la validez de contenido y de constructo y la confiabilidad, resultando ser un instrumento válido y fiable [5].

La encuesta fue aplicada en el período de marzo - abril del 2017 a los turistas internacionales que arriban al Salón de Primera Espera (antes de ingresar al destino Cuba) del Aeropuerto Internacional José Martí, se eligió este lugar porque estas personas aún no han interactuado con los servicios de restauración y es un momento oportuno para conocer lo que ellos esperan recibir del servicio de alimentos y bebidas ofrecido en los diferentes puntos de ventas del país.

Se escogió una muestra probabilística aleatoria, de 102 turistas, cantidad estimada para poblaciones infinitas (mayor de 100000 ), considerando un nivel de confianza del $95 \%(\alpha$ $=0,05)$, un error muestral del $9 \%$. Se utilizó para esto la calculadora en Excel para tamaño muestral.

El cuestionario está conformado por 13 preguntas multidimensionales, con respuestas tanto abiertas como cerradas. En el caso de las cerradas, estas se presentaron de las tres maneras existentes según Ruiz, (2002): dicotómicas (2 alternativas de respuesta-sí/no), policotómicas (varias opciones de respuesta) y analógica verbal (escala de tipo Likert) [6].

Los datos obtenidos fueron procesados mediante el método porcentual a partir del cálculo de la frecuencia de las respuestas emitidas por los encuestados. Se utilizó el programa estadístico "Statistic Program for Social Sciences" (SPSS) para Windows (versión 22.0).

Con el objetivo de determinar si existe diferencia significativa entre las prioridades de los clientes para la selección de los restaurantes, se empleó la prueba no paramétrica de ordenamiento por rangos, procesando los resultados mediante el análisis bidimensional de Friedman considerando un nivel de confianza del 95\% $(\alpha<0.05)$.

Se fijaron las hipótesis siguientes:

Hipótesis nula. Ho: La distribución de las prioridades para la selección del restaurante son iguales.

Hipótesis alternativa. H1: La distribución de las prioridades para la selección del restaurante son significativamente diferentes.

Cuando se cumplió la hipótesis alternativa (H1) se realizó la prueba de Wilcoxon para determinar entre cuáles de las variables analizadas existió diferencia significativa para un nivel de confianza del $95 \%$ según reporta Espinosa (2015) [7].

Se empleó el Diagrama de Pareto para el procesamiento de la pregunta abierta con el propósito de determinar los principales servicios adicionales que los turistas desean recibir en un restaurante.

La determinación de la confiabilidad del instrumento, se realizó a partir del coeficiente Alfa de Cronbach. Se consideró lo reportado por Hernández, Fernández y Baptista, 
(2010), que establecen que: si el valor del coeficiente supera el 0.75 es aceptable, y si es mayor a 0.90 es elevada [8].

\section{Resultados.}

- Datos demográficos de los turistas encuestados

La muestra de turistas encuestados se caracterizó por ser en su mayoría del género femenino $(51,8 \%)$. En cuanto a la edad, el rango predominante $(40,2 \%)$ fue de $26-40$ años, el $27,8 \%$ corresponde a personas entre 41 y 60 años, representando estos dos grupos el $68 \%$ de la muestra. En cuanto a la nacionalidad, se aprecia que los estadounidenses $(28,4 \%)$ son los que priman, seguido por los mexicanos con una representación de un $20 \%$, luego los españoles y los franceses con un valor porcentual de $8,4 \%$ y $7,4 \%$ respectivamente.

La mayor cantidad de los turistas encuestados $(65,7 \%)$ declararon haber viajado a Cuba por primera vez, mientras que el $34,3 \%$ declara haber venido en otras ocasiones. De este último, el $75 \%$ expresa que sus expectativas se cumplieron en relación al servicio de alimentos y bebidas recibido. Los planteamientos que más repercuten en el no cumplimiento de sus expectativas corresponden a los precios elevados de la oferta gastronómica, la poca variedad de la misma y el mal servicio recibido, con valores de $26,3 \% ; 21,1 \%$ y $15,8 \%$ respectivamente.

- Resultados de las expectativas de los turistas sobre las dimensiones "Oferta gastronómica", "Personal" e "Instalación".

En la figura 1 se presentan los resultados con respecto a las dimensiones "Oferta gastronómica", "Personal" e "Instalación".

Figura 1. Expectativas de los turistas sobre las dimensiones "Oferta gastronómica", "Personal" e "Instalación".

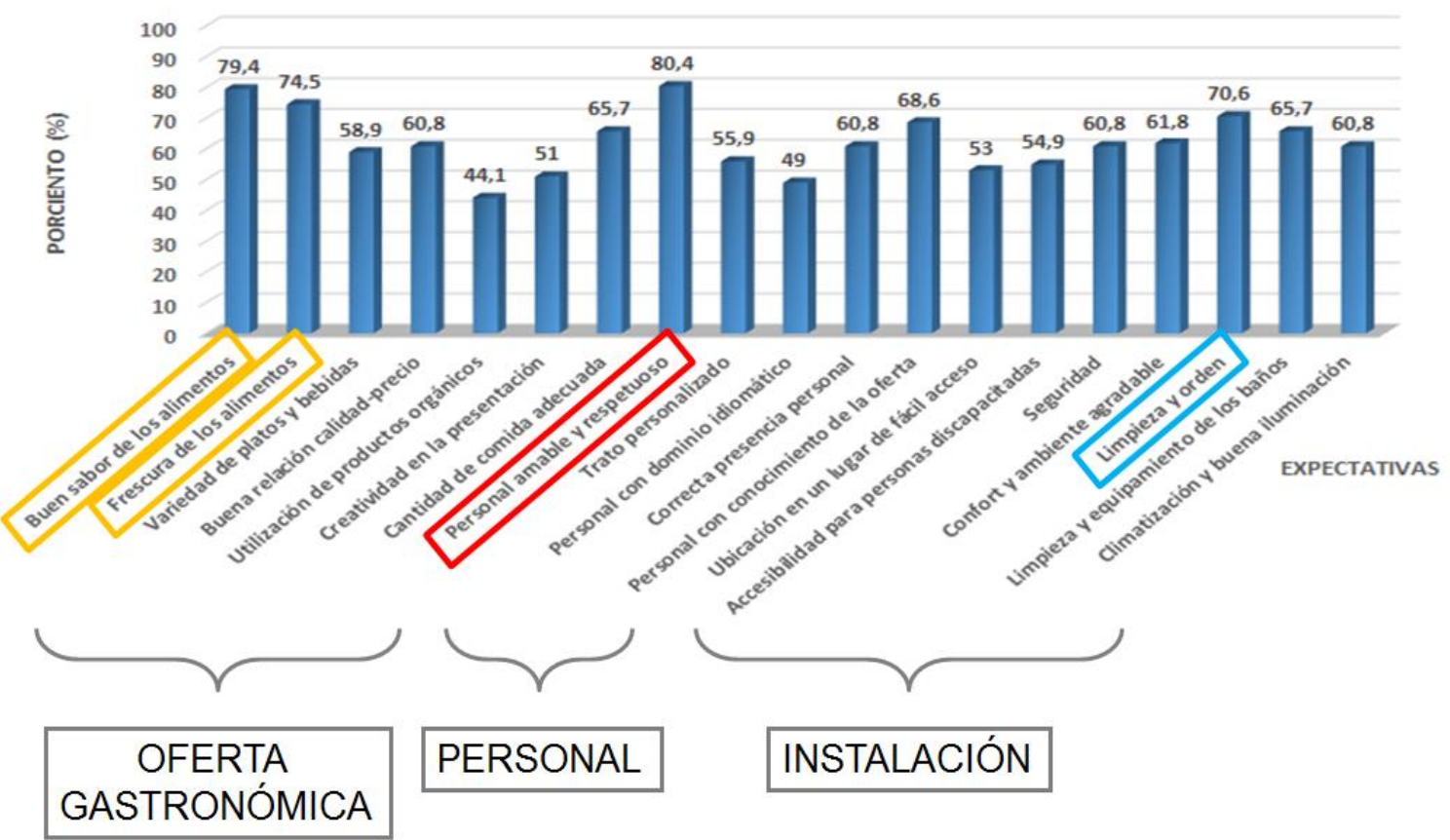


Como se puede observar, dentro de la oferta gastronómica las variables más valoradas y declaradas como las que generan mayor expectación a los turistas son "Buen sabor de los alimentos" (79,4\%), "Frescura de los alimentos" (74,5\%), "Cantidad de comida adecuada" $(65,7 \%)$ y "Buena relación calidad-precio" con un $60,8 \%$. El ítem sobre el cual se tiene menos expectativa es "Utilización de productos orgánicos" con un valor de 44,1\%.Tres indicadores alcanzan el mayor porcentaje de expectativas en relación al personal de contacto, tal es el caso de "Personal amable y respetuoso" $(80,4 \%)$ que ocupa el primer lugar, le sigue "Personal con conocimiento de la oferta" $(68,6 \%)$ y luego el atributo "Correcta presencia personal" (60.8\%).

En el caso de los indicadores concernientes a la dimensión "Instalación" la "Limpieza y orden" (70,6\%), "Limpieza y equipamiento de los baños" $(65,7 \%)$ son los que reportaron mayores porcentajes de expectativas, seguido de "Confort y ambiente agradable" con un valor porcentual de $61,8 \%$. Estos ítems son vitales en el acondicionamiento del local. Más de la mitad de la muestra (54,9\%) desea que exista accesibilidad para personas discapacitadas y que los restaurantes se ubiquen en lugares accesibles (53\%).

La figura 2 muestra el porcentaje de satisfacción de las expectativas en relación al servicio de alimentos y bebidas en otros países visitados por los turistas, si bien esta interrogante no tributa de manera directa al fenómeno estudiado resulta oportuno disponer de esta información. Como se puede observar los países más visitados por los turistas son Estados Unidos, Italia, Francia, España y México con un valor de 54,9\%; 47,1\%; 44,1\%; 43,1\% y 35,3\% respectivamente, según OMT (2016) estos países están incluidos en el ranking de los diez mayores destinos del mundo en cuanto a llegadas de turistas [9].

Figura 2. Porcentaje de satisfacción de las expectativas en relación al servicio de alimentos y bebidas en otros países visitados por los turistas.

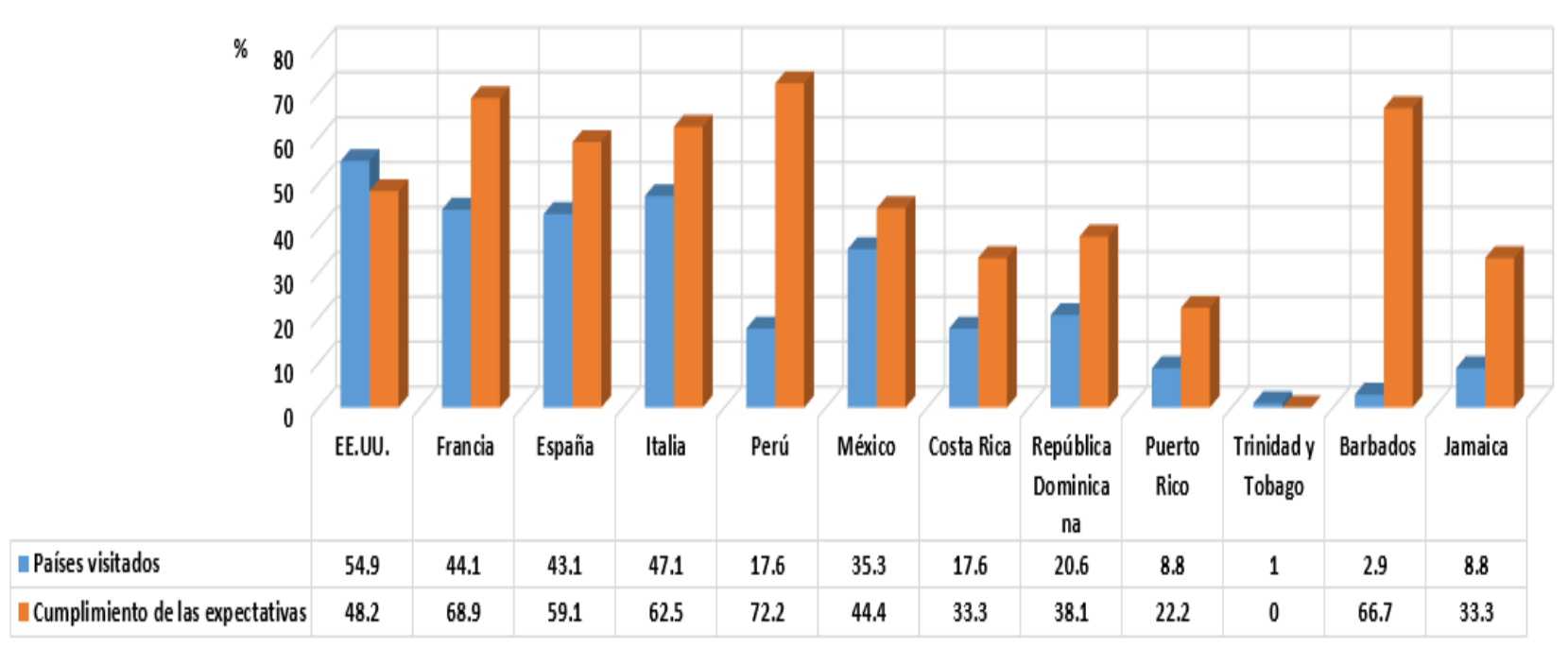

La tabla 1 muestra las prioridades de los turistas cuando selecciona un restaurante, se evidencia que la "comida", constituye lo más importante, estableciendo una diferencia significativa $(\mathrm{p}<0.05)$ con respecto al resto de las variables. La "seguridad" y el "precio" se ubican en el segundo lugar con un mismo orden de prioridad. No existe diferencia 
significativa entre los variables "servicio gastronómico" "personal" e "instalación", que ocupan el tercer lugar, mientras que la accesibilidad y la sostenibilidad resultaron ser las de menor prioridad.

Tabla 1. Prioridades de los turistas a la hora de seleccionar un restaurante

\begin{tabular}{|l|c|c|}
\hline \multicolumn{1}{|c|}{ Variables } & Sumatoria & \\
\hline Comida & 97 & $\mathrm{a}$ \\
\hline Seguridad & 150 & $\mathrm{~b}$ \\
\hline Precio & 162 & $\mathrm{~b}$ \\
\hline S. Gastronómico & 376 & $\mathrm{c}$ \\
\hline Personal & 400 & $\mathrm{c}$ \\
\hline Instalación & 462 & $\mathrm{c}$ \\
\hline Accesibilidad & 630 & $\mathrm{~d}$ \\
\hline Sostenibilidad & 696 & $\mathrm{~d}$ \\
\hline
\end{tabular}

Letras iguales indican que no existe diferencia significativa para $p<0.05$

En la figura 3 es posible apreciar que 6 aspectos de los 10 analizados constituyen los servicios vitales deseados por los turistas en un restaurante, representando un $79 \%$ del total de servicios adicionales, estos son: música agradable, servicio de atención al cliente, servicio de información sobre la especialidad de la casa, servicio de internet, servicio de animación y servicio de seguridad.

Figura 3. Diagrama de Pareto. Servicios adicionales que los turistas desean recibir en un restaurante.

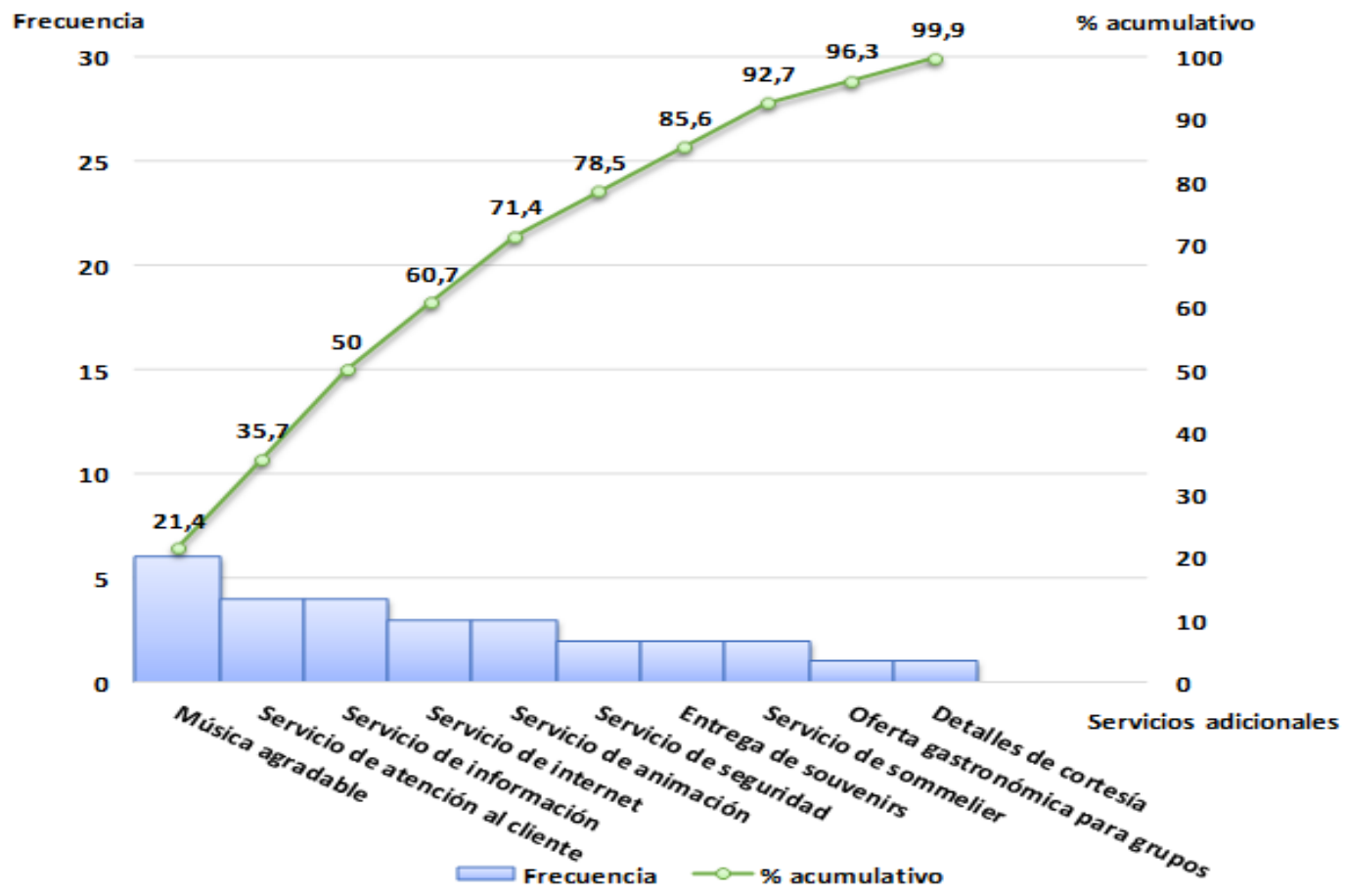


- Determinación de la confiabilidad del instrumento.

Los niveles de confiabilidad, obtenidos con el método de Alfa de Cronbach en cada una de las dimensiones y en el cuestionario de manera general, oscilaron entre 0,886 y 0,937; tal como puede observarse en la tabla 2, siendo en todos los casos resultados muy favorables, ya que reflejaron un alto índice de consistencia interna.

Tabla 2. Resultados del coeficiente $\alpha$ de Cronbach.

\begin{tabular}{|l|c|}
\hline \multicolumn{1}{|c|}{ Indicadores } & Consistencia interna \\
\hline Instalación & 0,937 \\
\hline Personal & 0,875 \\
\hline $\begin{array}{l}\text { Oferta } \\
\text { gastronómica }\end{array}$ & 0,886 \\
\hline $\begin{array}{l}\text { Cuestionario } \\
\text { general }\end{array}$ & 0,923 \\
\hline
\end{tabular}

\section{Discusión.}

Al analizar la proyección de las expectativas de los turistas con las dimensiones "Oferta gastronómica", "Personal" e "Instalación", se fundamenta la necesidad de prestar especial atención al sabor y a la frescura ya que resultaron ser variables sobre las cuales se tienen mayores expectativas. En relación a ello, Moubarac, (2015) apunta que una alimentación saludable se basa en comidas frescas y manufacturadas, derivadas de sistemas alimentarios sostenibles y culturas alimentarias establecidas. La alimentación que está compuesta en su mayor parte por productos ultra procesados listos para consumir, provenientes del sistema alimentario industrial globalizado (comida rápida), no es saludable. [10].

Por otra parte se evidenció que los turistas esperan en mayor medida ser tratados por un personal amable, conocedor de las normas de cortesía y educación, con conocimiento de la cultura gastronómica y de la oferta del menú, con una correcta presencia personal, que sea capaz de escucharlos y comprender sus necesidades y deseos.

Varela [et al.] (2006) plantea que diversos estudios destacan la importancia que tiene el atributo "Limpieza y equipamiento de los baños" en la satisfacción de las expectativas de los turistas con respecto a la instalación, no sólo por la higiene y su equipamiento, sino también por su capacidad [11].

En cuanto a la satisfacción de las expectativas en relación al servicio de alimentos y bebidas en otros países visitados por los turistas, se debe señalar que las encuestas revelaron que Perú, Francia, España e Italia, destacan con valores elevados de satisfacción. Lo cual es comprensible ya que estos países son de reconocida trayectoria gastronómica, de ahí que constituyen referentes obligados en cuanto a buenas prácticas en la restauración.

Es menester destacar como el cliente valora la "seguridad" dentro de las principales prioridades, según la Organización Mundial del Turismo (2016) la seguridad es un factor subyacente de la calidad [13]. En el marco de los servicios de alimentos y bebidas esto se 
traduce también en garantía de la inocuidad a partir de la implementación de las buenas prácticas de elaboración de alimentos.

Sin embargo, llama la atención que a pesar de que en la actualidad una de las tendencias es la sostenibilidad en todos los ámbitos del sector y de la sociedad; aún no se visualiza en su totalidad la importancia de esta variable y no constituye todavía una prioridad a la hora de visitar el restaurante, esto habla de la necesidad de promover en el cliente una conciencia medioambiental y el compromiso con la sostenibilidad.

Es preciso tener en cuenta que los servicios adicionales resultan claves, son elementos diferenciadores de la oferta y aportan el valor añadido a los clientes, en el ámbito de la restauración se trata de generar experiencias agradables y memorables; y también facilitar las actividades de los clientes. A decir de los encuestados el abanico de servicios complementarios identificados como vitales se desplaza desde aspectos tradicionales y manidos como la música hasta el servicio de internet, indispensable en el mundo actual. Cabe subrayar que dentro de estos servicios se encuentra nuevamente el indicador de seguridad, lo que se corresponde con los resultados obtenidos previamente.

La réplica de este estudio y ampliar el tamaño de muestra es primordial para dar continuidad a la investigación. A partir de lo expresado, resulta oportuno señalar que garantizar la prestación de un servicio de alimentos y bebidas con calidad que satisfaga las expectativas de los clientes, contribuirá al incremento de la competitividad del destino Cuba.

\section{Conclusiones.}

Se demostró que los atributos "Personal amable y respetuoso, el "Buen sabor de los alimentos", la "Frescura de los alimentos y la "Higiene y el orden" son las variables de mayor expectación para los turistas, siendo la "Utilización de productos orgánicos" la que genera menor expectativa.

Los indicadores más importantes a la hora de seleccionar un restaurante son: la comida, la seguridad y el precio, mientras que la accesibilidad y la sostenibilidad resultaron ser los de menor prioridad.

Los servicios adicionales vitales que los turistas esperan encontrar en el destino son: música agradable, servicio de atención al cliente, servicio de información sobre la especialidad de la casa, servicio de internet, servicio de animación y servicio de seguridad.

\section{Referencias Bibliográficas.}

ORGANIZACIÓN MUNDIAL DEL TURISMO. "Panorama del turismo internacional. Informe Anual”. Edición 2017. ISBN: 978-92-844-1904-3

MESA RAMOS, Lideimys. "Diseño de un instrumento para la determinación de las expectativas de los turistas sobre el servicio de alimentos y bebidas en el destino 
Cuba". Tesis de Licenciatura. Facultad de Turismo. Universidad de La Habana. 2017. 65 p.

CALÁS FERNÁNDEZ, Dayamí. Validación del cuestionario para la evaluación de la percepción de la calidad del servicio de alimentos y bebidas en los restaurantes de la red hotelera y extrahotelera del Ministerio de Turismo. Tesis de Licenciatura. Facultad de Turismo. Universidad de La Habana. 2014. 62 p.

MORENO, Mayra Rosario. [et al.] La gestión de la calidad en el proceso de manejo de quejas en el área de restauración "Contribuciones a la Economía". Revista académica. 2012. ISSN 1696-8360

ROMANÍ BENDIG, Beatriz. [et al.] Diseño de un instrumento para la determinación de las expectativas de los turistas sobre el servicio de alimentos y bebidas en el destino Cuba. En XIV Conferencia Internacional de Ciencia y Tecnología de los Alimentos. (2018. Cuba) 13 p. ISBN: 978-959-7003-52-6

RUIZ BOLÍVAR, C. Instrumentos de Investigación Educativa. Procedimiento para su Diseño y Validación. Venezuela: CIDEC. 2002. Edición 1ra. ISBN 980-303-3840

ESPINOSA MANFUGÁS, J.M. Evaluación Sensorial. Editorial Félix Varela La Habana. Cuba, 2015. ISBN 978-959-07-1956-1

HERNÁNDEZ, R.; FERNÁNDEZ, C. y BAPTISTA, M. P. Metodología de la investigación (5ta ed.). Editorial McGraw Hill Interamericana de México, S.A. 2010. ISBN: 978-607-15-0291-9

ORGANIZACIÓN MUNDIAL DEL TURISMO. "Panorama del turismo internacional. Informe Anual". Edición 2016.

MOUBARAC, J. C. Alimentos y bebidas ultra procesados en América Latina: tendencias, efecto sobre la obesidad e implicaciones para las políticas públicas. Departamento de Enfermedades no Transmisibles y Salud Mental. Washington, DC: Organización Panamericana de la Salud, 2015. ISBN 978-92-75-31864-5

VARELA, MALLOU. Jesús [et al.] Una nueva escala para la evaluación de la calidad de los servicios de hostelería. Revista Psicothema, 18 (1): 135-142, 206, 2006. ISSN 0214-9915

ORGANIZACIÓN MUNDIAL DEL TURISMO. La gestión de la calidad, esencial para la competitividad de los destinos turísticos. Comité de Turismo y Competitividad de la OMT. PR No.: 16052. [en línea] Disponible en: http://www.worldtourism.org/quality [Consultado el 8 de febrero 2018] 


\section{Para citar el artículo indexado.}

Romaní B., Espinosa J., Tejedor R. \& Mesa L. (2019). Expectativas del turista con el servicio de alimentos y bebidas en Cuba. Revista electrónica Explorador Digital 3(2), 1625. Recuperado desde:

http://cienciadigital.org/revistacienciadigital2/index.php/exploradordigital/article/view/416/9 $\underline{47}$

\section{Ciencia \\ Digital \\ Editorial}

El artículo que se publica es de exclusiva responsabilidad de los autores y no necesariamente reflejan el pensamiento de la Revista Explorador Digital.

El articulo queda en propiedad de la revista y, por tanto, su publicación parcial y/o total en otro medio tiene que ser autorizado por el director o editor de la Revista Explorador Digital.
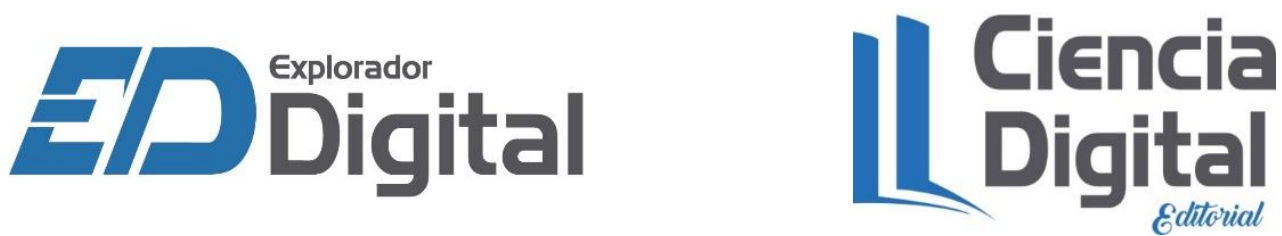